\title{
Formononetin Suppresses Interleukin-6 Production in Lipopolysaccharide-Stimulated Macrophages Via Activation of the AMP-Activated Protein Kinase
}

\section{Lanfang Zhang}

Shanxi University of Traditional Chinese Medicine

Xiaoyan Zhang

Fenyang College Shanxi Medical University

Liying Wang

Shanxi University of Traditional Chinese Medicine

Wanxia Yang

Fenyang College Shanxi Medical University

Yuanbiao Qiao

Shanxi University of Traditional Chinese Medicine

Qingshan Li ( $\nabla$ sxlqs2012@163.com )

\section{Research}

Keywords: Lipopolysaccharide, Interleukin-6, AMP-activated protein kinase, formononetin, macrophage

Posted Date: July 29th, 2020

DOI: https://doi.org/10.21203/rs.3.rs-48445/v1

License: (c) (1) This work is licensed under a Creative Commons Attribution 4.0 International License. Read Full License 


\section{Abstract}

Background: Interleukin-6 (IL-6), an inflammatory cytokine, plays a majoy role in the pathogenesis of inflammation and serves as a key marker in the diagnosis and treatment of inflammation and related diseases. Formononetin (FMN), an isoflavone ingredient in various Chinese herbal medicines, inhibits the production of IL-6 in some cells. However, its mechanism of action has not been clearly established. In this study, we aimed to identify whether FMN inhibits IL-6 production in Lipopolysaccharide (LPS)stimulated macrophages via AMPK activation.

Methods: In the current study, the anti-IL-6 mechanism of FMN was evaluated using a macrophage model with LPS stimulation. An inverted microscope was utilized to obtain images of cells. Nuclear staining assay and CCK-8 assay were used to identify the viability of ANA-1 cells. The expression of IL- 6 in cells was investigated by Enzyme-linked immunosorbent (ELISA) and Western blotting. The expression of AMP-activated protein kinase (AMPK) was determined by Western blotting and phosphorylation of AMPK was determined by Western blotting and Immunofluorescence assay. Bioinformatic analysis was used to predict potential targets of FMN.

Results: we found that FMN reduced the expression of IL-6 in ANA-1 cells and increased the phosphorylation of AMPK. The effect of FMN was similar to that of acadesine, an AMPK activator, which also reduced IL- 6 expression in LPS-induced ANA-1 cells and increased AMPK phosphorylation. Its combination with dorsomorphin (an AMPK inhibitor), however, reversed the effects of FMN on AMPK phosphorylation and IL-6 expression. The target of FMN was identified as the cAMP-dependent protein kinase inhibitor alpha, as searched in Traditional Chinese Medicine Systems Pharmacology Database and Analysis Platform and further verified with the STRING database.

Conclusions: In conclusion, our findings suggest that FMN effectively inhibits IL-6 production by activating AMPK in LPS-stimulated macrophages.

\section{Introduction}

Interleukin-6 (IL-6), an important inflammatory cytokine, plays various roles in the human body and is involved in different inflammatory diseases. Hence, it is considered a therapeutic target for such diseases [1-3]. As a response to infection or tissue damage, macrophages immediately produce IL-6 via the activation of pattern recognition receptors. Lipopolysaccharide (LPS), which is a bacterial endotoxin present in the cell wall of gram-negative bacteria, can induce an inflammatory response in the body, causing injury, disease, and even death [4-6]. The uncontrolled excess or sustained production of IL-6 through LPS induction can lead to the development of various inflammatory diseases and cancers [7]. IL6 expression is mainly associated with the activation of nuclear factor-KB (NF-KB), nuclear factor-IL-6, tumor-necrosis factor, and other factors $[8,9]$. The conventional anti-inflammatory drugs currently used have various adverse effects; therefore, it is imperative to find new anti-IL-6 drugs. 
Formononetin (FMN) is a typical isoflavone compound. It is extracted from herbs used in traditional Chinese medicines, such as Astragalus membranaceus [10] and Trifolium pratense L. [11]. The molecular formula of $F M N$ is $\mathrm{C}_{16} \mathrm{H}_{12} \mathrm{O}_{4}$. Its structural formula is shown in Fig. 1. Some studies have shown that FMN has various pharmacological effects such as anti-cancer, anti-oxidation, and anti-inflammatory [12, 13]. Moreover, FMN can inhibit LPS-induced IL-6 expression in cells including macrophages [14], microglia [15], and bone marrow-derived dendritic cells [16]. However, the mechanism of its action is not completely clear; the upstream signaling pathways and targets thus require further research.

Studies have reported that FMN can activate 5 ' AMP-activated protein kinase (AMPK) $[17,18]$, which is a key regulator protease commonly expressed in cells to provide energy. Cells activate AMPK via adenine nucleotide-dependent and other non-classical pathways to meet their energy requirements [19]. Studies have demonstrated that AMPK, which is located upstream of multiple signaling pathways, can inhibit IL-6 expression [20, 21]. However, whether FMN exerts an anti-IL-6 production effect via AMPK remains unclear. In this study, we aimed to identify whether FMN inhibits IL-6 production in LPS-stimulated macrophages via AMPK activation.

\section{Materials And Methods}

\section{Drugs and chemicals}

FMN (YZ-1248S), LPS (L8880), and dimethyl sulfoxide (DMSO, D8371) were purchased from Solarbio (Beijing, China). Triton X-100 was obtained from Sigma. Acadesine (S1515), Western and IP cell lysates (P0013), and Mouse IL-6 ELISA Kit (PI326) were purchased from Beyotime Biotechnology (Shanghai, China). Dorsomorphin (P5499) was purchased from Sigma-Aldrich. The Anti-IL-6 Rabbit pAb (GB11117) was obtained from Servicebio (Wuhan, China). Phospho-AMPK (p-AMPK) alpha antibody (Thr172) (AF3423), AMPK alpha 1 antibody (BF2001), and Goat Anti-Rabbit IgG $(\mathrm{H}+\mathrm{L})$ HRP antibody (S0001) were obtained from Affinity Biosciences (Ohio, USA). The $\beta$-Actin antibody (AP0060) was obtained from Bioworld (Bloomington, USA). IRDye 680LT Goat anti-Rabbit IgG (925-68021) and IRDye 800CW goat antimouse IgG $(\mathrm{H}+\mathrm{L})$ antibody (926-32210) were obtained from LICOR (Nebraska, USA). TureColor two-color pre-stained protein marker (C520010-0250) was purchased from Sangon Biotech (Shanghai, China) and CellMax Fetal Bovine Serum (SA211.02) from CellMax (Beijing, China). RPMI 1640 Medium (42401042) was obtained from Thermo Fisher (Waltham, USA). Cell Counting Kit-8 (CCK-8, AR1199), 4\% paraformaldehyde (AR1068), 4',6-diamidino-2-phenylindole (DAPI, AR1176), BCA Protein Quantification kit (AR0146), $5 \times$ loading buffer (AR1112-10), DyLight 488 anti-rabbit IgG (BA1127), and ECL Western Blot Detection Kit (AR1170) were purchased from Boster Biotechnology (California, USA).

\section{Cell culture and morphological observations}

ANA-1 cells were purchased from the Shanghai Institute of Cell Biology, Chinese Academy of Sciences (Shanghai, China) and cultured in 1640 medium at $37^{\circ} \mathrm{C}$ in a $5 \%$ humidified $\mathrm{CO}_{2}$ incubator. At intervals of $24 \mathrm{~h}$, the cells were passaged and observed with an inverted microscope. 


\section{Nuclear staining assay}

ANA-1 cells were stimulated with LPS at varying concentrations for $24 \mathrm{~h}$. The cells were fixed with $4 \%$ paraformaldehyde for $1 \mathrm{~h}$ and then permeabilized with 1\% Triton X-100 for 30 min and blocked with 1\% BSA in phosphate-buffered saline (PBS) for $60 \mathrm{~min}$. The cells were stained with DAPI for $10 \mathrm{~min}$ and washed three times with PBS between each step. An inverted microscope was used to obtain images of cells at $20 \times$ magnification and ImageXpress micro, a high-content imaging analysis system (HCS), was used to determine the total cell count in each LPS-stimulated group.

\section{CCK-8 assay}

ANA-1 cells in the logarithmic growth phase were inoculated in 96-well plates. The cells were treated with equal volumes of DMSO solvent, the LPS solution, FMN solution, and the LPS + FMN solution for $24 \mathrm{~h}$. Clear medium without cells was used as a blank and the untreated cells were the control. Then, $10 \mu \mathrm{L}$ of CCK-8 reagent was added into each well and incubated at $37^{\circ} \mathrm{C}$ for $1.5 \mathrm{~h}$. A microplate reader was used to measure the absorbance of the sample in each well at $450 \mathrm{~nm}$, and the proliferating percentage of cells was calculated as follows:

Cell proliferation percentage $(\%)=[$ (experimental group absorbance - blank group absorbance) $/$ (control group absorbance - blank group absorbance) $] \times 100 \%$

\section{Enzyme-linked immunosorbent assay (ELISA)}

ANA-1 cells were exposed to LPS and FMN at different concentrations for $24 \mathrm{~h}$. The levels of IL-6 in ANA1 cells supernatants were quantified with commercially available ELISA kits (Beyotime Biotechnology, China), following the company's directions.

\section{Western blotting}

The treated cells were collected at the indicated time points and lysed with cell lysis buffer containing protease inhibitors. The total protein was quantified using the BCA method. The total proteins were subjected to $12 \%$ SDS-PAGE and transferred onto nitrocellulose (NC) membranes. The NC membranes were incubated with $\beta$-actin (1:1500), IL-6 (1:1000), p-AMPK (1:1000), and AMPK (1:1000) antibodies at $4{ }^{\circ} \mathrm{C}$ overnight. After three washes, the NC membrane was incubated with the HRP-labeled secondary antibodies (1:5000), IRDye 680LT Goat anti-Rabbit IgG (1:20000), or IRDye 800CW goat anti-mouse IgG $(\mathrm{H}+\mathrm{L})$ antibody $(1: 15000)$ at $20^{\circ} \mathrm{C}$ for $2 \mathrm{~h}$. The blot was then developed with a sensitive ECL chemiluminescent substrate and detected using a chemiluminescent gel imager [22]. After washing, another NC membrane with infrared fluorescently labeled secondary antibodies was scanned using a near-infrared laser imager Amersham Typhoon NIR. Finally, the results were quantitatively analyzed using Image J software.

\section{Immunofluorescence assay}


ANA-1 cells in the logarithmic growth phase were inoculated in 96-well plates. The cells were treated with FMN solution and the LPS + FMN solution for $24 \mathrm{~h}$. The cells were fixed with $4 \%$ paraformaldehyde for $1 \mathrm{~h}$ and then permeabilized with 1\% Triton X-100 for 30 min and blocked with 1\% BSA in PBS for 60 min. Cells were then incubated with p-AMPK $(1: 200)$ antibodies at $4{ }^{\circ} \mathrm{C}$ overnight. Next, cells were incubated with DyLight 488 anti-rabbit $\lg G(1: 500)$ for $2 \mathrm{~h}$ at $20^{\circ} \mathrm{C}$. The cells were washed three times with PBS between each step. Then, the HCS was used to obtain images of cells at $200 \times$ magnification and determine the cell average FITC intensity in each group.

\section{Bioinformatic analysis}

The Computational Systems Biology Laboratory (TCMSP - Traditional Chinese Medicine Systems Pharmacology Database and Analysis Platform, https://tcmspw.com/tcmsp.php) database was used to search potential targets of FMN, and the STRING website (STRING - functional protein association networks, https://string-db.org/) was used to analyze protein interactions.

\section{Statistical analysis}

All experiments were repeated at least three times. The data were analyzed using the one-way ANOVA and Student's $t$-test with GraphPad Prism 7.04 software. Results with a $p$ value $<0.05$ were considered statistically significant.

\section{Results}

\section{LPS increases IL-6 levels in ANA-1 cells}

To determine whether the proliferation of ANA-1 cells is inhibited by LPS, the cells were treated with LPS $(0.05,0.1,0.5,1$, and $2 \mu \mathrm{g} / \mathrm{mL})$ for $24 \mathrm{~h}$ and evaluated using the HCS. ANA-1 cells were observed under an inverted microscope and appeared to have an irregular shape with enlargement, protrusion, and film-like pseudopods (Fig. 2a). The total cell count decreased as the concentration of LPS increased (Fig. 2b). In addition, the results revealed that the incubation of ANA-1 cells with varying concentrations of LPS for $24 \mathrm{~h}$ increased IL-6 expression in a dose-dependent manner (Fig. 2c-2e). The above results suggest that LPS is toxic to ANA-1 cells and can induce ANA-1 cells to produce excessive amounts of IL-6.

\section{FMN attenuates LPS-induced toxicity in ANA-1 cells}

To determine whether FMN affects cell proliferation, the cells were treated with FMN at different concentrations $(0.016,0.08,0.4,2,10$, and $50 \mu \mathrm{mol} / \mathrm{mL})$ for $24 \mathrm{~h}$, and the cell proliferation rate was determined using the CCK-8 kit. Compared to that in the control group, an increase in the FMN concentration did not affect the cell proliferation rate. Furthermore, there was no significant difference between the DMSO solvent and control groups (Fig. $3 \mathrm{a}$ ). On the other hand, the proliferation rate of LPSstimulated cells was increased with FMN treatment at varying concentrations $(0.08,0.4,2,10$, and $50 \mu \mathrm{mol} / \mathrm{mL}$ ) for $24 \mathrm{~h}$, and the result was statistically significant at the concentrations of 10 and $50 \mu \mathrm{mol} / \mathrm{mL}$ FMN (Fig. 3b). 


\section{FMN reduces IL-6 levels in LPS-stimulated ANA-1 cells}

We next investigated the effect of FMN on IL-6 expression induced by LPS. LPS-stimulated ANA-1 cells were exposed to FMN at $0.4,2$, and $10 \mu \mathrm{mol} / \mathrm{mL}$ concentrations for $24 \mathrm{~h}$. We found that FMN decreased the IL-6 levels in LPS-stimulated cells in a dose-dependent manner (Fig. 4), confirming that FMN can inhibit IL-6 production.

\section{FMN increases the phosphorylation of AMPK in ANA-1 cells}

We then explored how FMN inhibits IL-6 expression. AMPK, a potent inhibitor of IL- 6 expression, is present upstream of multiple signaling pathways. We investigated the regulatory effect on AMPK by exposing ANA- 1 cells to FMN at concentrations of $0.08,0.4,2$, and $10 \mu \mathrm{mol} / \mathrm{mL}$ for $24 \mathrm{~h}$. The western blotting results revealed that the $\mathrm{p}$-AMPK level in ANA-1 cells was increased with FMN treatment at concentrations of $0.4,2$, and $10 \mu \mathrm{mol} / \mathrm{mL}$. However, FMN has no effect on the expression levels of the total AMPK protein (Fig. 5a, 5b, and 5e). Furthermore, FMN dose-dependently increased the level of p-AMPK (Fig. 5c,5d, 5f, and $5 \mathrm{~g}$ ) in ANA-1 cells when they were co-treated with varying FMN concentrations $(0.4,2$, and $10 \mu \mathrm{mol} / \mathrm{mL}$ ) and $1 \mu \mathrm{g} / \mathrm{mL}$ LPS for $24 \mathrm{~h}$. These results suggest that FMN might inhibit IL-6 production by activating AMPK.

\section{Acadesine increases the phosphorylation of AMPK in ANA-1 cells and reduces the levels of IL-6}

Considering the close association between IL- 6 and AMPK, we explored the effect of acadesine (AICAR) on these two proteins. We found that AICAR at varying concentrations $(20,40$, and $80 \mu \mathrm{mol} / \mathrm{mL})$ increased the levels of p-AMPK in ANA-1 cells (Fig. 6a). Furthermore, treatment with 20, 40, and $80 \mu \mathrm{mol} / \mathrm{mL}$ AICAR for $24 \mathrm{~h}$ increased the levels of $\mathrm{p}$-AMPK compared to those with LPS treatment in ANA-1 cells and reduced the levels of IL-6 in a dose-dependent manner (Fig. 6c). Overall, the activation of AMPK can inhibit the production of IL-6 in LPS-stimulated ANA-1 cells.

Dorsomorphin reverses the effect of FMN on AMPK phosphorylation and IL-6 protein expression in LPSstimulated ANA- 1 cells

We confirmed the relationship between IL- 6 and p-AMPK using an AMPK inhibitor, dorsomorphin (compound C, CC). ANA-1 cells were treated with LPS, LPS + CC, LPS + FMN, and LPS + CC + FMN. Western blotting was performed to determine the levels of $\mathrm{p}-\mathrm{AMPK}$ and IL-6. Compared to that with LPS treatment, LPS + CC treatment decreased the p-AMPK levels and increased the IL- 6 levels in ANA-1 cells, whereas the LPS + FMN treatment increased the levels of p-AMPK and reduced the levels of IL- 6 in ANA-1 cells. Compared to that with LPS + FMN treatment, LPS + FMN + CC treatment suppressed p-AMPK expression and increased IL-6 expression simultaneously (Fig. 7a, 7b). These results suggest that the inhibitory effect of FMN on the production of IL- 6 in ANA- 1 cells was blocked by the inactivation of AMPK, indicating that AMPK signaling is involved in the inhibition of IL-6 production by FMN. 


\section{Prediction of the potential targets of FMN}

To elucidate the potential mechanism of action of FMN with respect to the activation of AMPK, we performed a bioinformatic analysis to search for potential targets of FMN. The TCMSP database search revealed that cAMP-dependent protein kinase inhibitor alpha (Pkia) is one of the potential targets of FMN. The analysis using the STRING database confirmed the CAMP-dependent protein kinase catalytic subunit alpha (Prkaca) mediated association between Pkia and AMPK, which is composed of 5'-AMP-activated protein kinase catalytic subunit alpha-1 (Prkaa1), 5'-AMP-activated protein kinase catalytic subunit alpha2 (Prkaa2), 5'-AMP-activated protein kinase subunit beta-1 (Prkab1), 5'-AMP-activated protein kinase subunit beta-2 (Prkab2), 5'-AMP-activated protein kinase subunit gamma-1 (Prkag1), 5'-AMP-activated protein kinase subunit gamma-2 (Prkag2), and 5'-AMP-activated protein kinase subunit gamma-3 (Prkag3) (Fig. 8).

\section{Discussion}

In this study, we found that FMN, an isoflavone compound found in many Chinese herbal medicines, reduced the cytotoxicity and IL- 6 production in the LPS-stimulated murine macrophage ANA-1 cells. The result was consistent with previous research on other types of cells [14-16]. Previous studies have shown that FMN can exert anti-inflammatory effects via the NF-KB signaling pathway [23], the cytokine-activated Janus kinase-signal transducer and activator of transcription (JAK-STAT) signaling pathway [24], and the nuclear factor erythroid 2-related factor/heme oxygenase-1 signaling pathway [25], among others. In this study, we sought to explore the underlying molecular mechanism of the cytoprotective effect of FMN in the presence of LPS and anticipated more novel and promising discoveries using this experimental model. The inhibition of IL-6 production was reported to occur via AMPK activation [20, 21]. We found that FMN increased AMPK phosphorylation in a dose-dependent manner in the model. To validate our results, the inhibitory role of AMPK in IL-6 production was confirmed with an activator of AMPK, acadesine. Furthermore, we performed an inhibition experiment with a specific AMPK inhibitor, dorsomorphin. As expected, the inhibitory effect of FMN on IL-6 production was attenuated by the addition of the AMPK inhibitor.

Overall, we have demonstrated that FMN inhibits IL- 6 production by activating AMPK. To determine whether FMN directly activates AMPK, we used the TCMSP database to search potential targets of FMN and found no evidence of a direct effect of FMN on AMPK. We found that Pkia might serve as a potential target of FMN and then confirmed the association between Pkia and AMPK. Furthermore, Pkia was reported as a specific inhibitor of the cyclic-AMP-dependent protein kinase A (PKA). Several studies have confirmed that PKA can activate AMPK $[26,27]$. Therefore, we speculated that FMN reduced the inhibitory effect on PKA by binding to Pkia (a way to consume Pkia) and promoted AMPK activation via the PKA pathway.

IL-6 is a type of cytokine mainly secreted by macrophages and is known to play an important role in immune regulation and inflammation. Inflammation plays a key role in the pathogenesis of various 
human chronic diseases [28] such as cancer [29], diabetes [30], cardiovascular diseases [31], and metabolic diseases [17, 32]. Upon inflammatory stimulus, a large amount of IL-6 is released. IL-6 is a proinflammatory cytokine that can cause immune disorders and amplify inflammation. Thus, blocking IL6 could be exploited as a new treatment strategy for inflammatory diseases [33]. FMN certainly deserves attention and has great therapeutic potential because of its effective inhibitory effect on IL- 6 generation in this study.

Furthermore, many studies suggest that AMPK is a potential therapeutic target for inflammation, cancer, diabetes, and other diseases [34-37]. We proposed that FMN, an activator of AMPK, could not only serve as a candidate drug for the treatment of IL-6-mediated diseases but might also be a novel therapeutic agent against other diseases in which AMPK activity represents a potential therapeutic strategy. Therefore, in future studies, we aim to identify the targets of FMN that are involved in the activation of AMPK and verify the anti-inflammatory effect of FMN using animal models.

\section{Conclusions}

In conclusion, we found that FMN inhibits IL- 6 production by activating the AMPK signaling pathway in LPS-induced macrophages. Our study provides an experimental basis and a theoretical explanation of the anti-inflammatory mechanism of FMN. Furthermore, FMN, an effective AMPK activator, is proposed to be a candidate drug for the treatment of IL-6-mediated diseases.

\section{Abbreviations}

FMN, formononetin; IL-6, interleukin-6; LPS, lipopolysaccharide; AMPK, 5' AMP-activated protein kinase; CC, compound C; DAPI, 4',6-diamidino-2-phenylindole; DMSO, dimethyl sulfoxide; ELISA, enzyme-linked immunosorbent assay; $\mathrm{HCS}$, high-content imaging analysis system; NF-kB, nuclear factor-kB; NC, nitrocellulose; p-AMPK, phospho-AMPK; PBS, phosphate-buffered saline; PKA, protein kinase A; Pkia, protein kinase inhibitor alpha; Prkaca, protein kinase catalytic subunit alpha; Prkab, 5'-AMP-activated protein kinase subunit beta; Prkag, 5'-AMP-activated protein kinase subunit gamma.

\section{Declarations}

\section{Declarations}

Ethics approval and consent to participate

Not applicable.

\section{Consent for publication}

Not applicable.

\section{Availability of data and materials}


Not applicable.

\section{Competing interests}

The authors declare that they have no competing interests.

\section{Funding}

This work was supported by the National Science and Technology Major Project of China (No. 2017ZX09101003-001-017); the National Natural Science Foundation of China (No. 81473100); and the Key Research and Development Plan (Key Project) of Shanxi Province (No. 201703D111033).

\section{Authors' contribution}

Lanfang Zhang and Xiaoyan Zhang contributed equally to this work.

Lanfang Zhang: Methodology, Data Curation, Writing - Original Draft Preparation.

Xiaoyan Zhang: Conceptualization, Methodology, Writing- Reviewing and Editing.

Liying Wang: Data Curation.

Wanxia Yang: Methodology.

Yuanbiao Qiao: Conceptualization, Reviewing and Editing.

Qingshan Li: Conceptualization, Reviewing and Editing, Project administration, Funding acquisition.

All authors read and approved the final manuscript.

\section{Acknowledgments}

The authors convey their thanks to Shanxi Key Laboratory of Innovative Drug for the Treatment of Serious Diseases Basing on the Chronic Inflammation.

\section{Authors' information}

${ }^{a}$ College of Traditional Chinese Medicines, Shanxi University of Traditional Chinese Medicine, Jinzhong, Shanxi 030619, P.R. China

${ }^{\mathrm{b}}$ Fenyang College of Shanxi Medical University, Fenyang, Shanxi032200, P.R. China

Authors e-mail address:

Lanfang Zhang: 1491549147@qq.com

Xiaoyan Zhang: 931326464@qq.com 
Liying Wang: 13652153045@163.com

Wanxia Yang: 1980831761@qq.com

Yuanbiao Qiao: qyb_0222@sina.com

Qingshan Li: sxlqs2012@163.com

*Contact details for the corresponding authors: Qingshan Li, College of Traditional Chinese Medicines, Shanxi University of Traditional Chinese Medicine, Jinzhong, Shanxi 030619, P.R. China. Tel/Fax: +8613834200504, e-mail: sxlqs2012@163.com.

\section{References}

1. Calabrese LH, Rose-John S. IL-6 biology: implications for clinical targeting in rheumatic disease. Nat Rev Rheumatol. 2014;10(12):720-7.

2. Jones $S A$, Jenkins BJ. Recent insights into targeting the IL-6 cytokine family in inflammatory diseases and cancer. Nat Rev Immunol. 2018;18(12):773-89.

3. Kang S, Tanaka T, Narazaki M, Kishimoto T. Targeting Interleukin-6 Signaling in Clinic. Immunity. 2019;50(4):1007-23.

4. Lampiasi N, Montana G. The molecular events behind ferulic acid mediated modulation of IL-6 expression in LPS-activated Raw 264.7 cells. Immunobiology. 2016;221(3):486-93.

5. Ren W, Hu L, Hua F, Jin J, Wang Y, Zhu L. Myeloid differentiation protein 2 silencing decreases LPSinduced cytokine production and TLR4/MyD88 pathway activity in alveolar macrophages. Immunol Lett. 2011;141(1):94-101.

6. Cheng N, Liang Y, Du X, Ye RD. Serum amyloid A promotes LPS clearance and suppresses LPSinduced inflammation and tissue injury. EMBO Rep 2018, 19(10).

7. Tanaka T, Narazaki M, Kishimoto T. IL-6 in inflammation, immunity, and disease. Cold Spring Harb Perspect Biol. 2014;6(10):a016295.

8. Tanaka T, Narazaki M, Masuda K, Kishimoto T. Regulation of IL-6 in Immunity and Diseases. Adv Exp Med Biol. 2016;941:79-88.

9. Hu SL, Huang CC, Tzeng TT, Liu SC, Tsai CH, Fong YC, Tang CH. S1P promotes IL-6 expression in osteoblasts through the PI3K, MEK/ERK and NF-kappaB signaling pathways. Int J Med Sci. 2020;17(9):1207-14.

10. Nie T, Zhao S, Mao L, Yang Y, Sun W, Lin X, Liu S, Li K, Sun Y, Li P, et al. The natural compound, formononetin, extracted from Astragalus membranaceus increases adipocyte thermogenesis by modulating PPARgamma activity. Br J Pharmacol. 2018;175(9):1439-50.

11. Mu H, Bai YH, Wang ST, Zhu ZM, Zhang YW: Research on antioxidant effects and estrogenic effect of formononetin from Trifolium pratense (red clover). Phytomedicine 2009, 16(4):314-319. 
12. Ong SKL, Shanmugam MK, Fan L, Fraser SE, Arfuso F, Ahn KS, Sethi G, Bishayee A. Focus on Formononetin: Anticancer Potential and Molecular Targets. Cancers (Basel) 2019, 11(5).

13. Tay KC, Tan LT, Chan CK, Hong SL, Chan KG, Yap WH, Pusparajah P, Lee LH, Goh BH. Formononetin: A Review of Its Anticancer Potentials and Mechanisms. Front Pharmacol. 2019;10:820.

14. Kim MS, Park JS, Chung YC, Jang S, Hyun CG, Kim SY. Anti-Inflammatory Effects of Formononetin 7O-phosphate, a Novel Biorenovation Product, on LPS-Stimulated RAW 264.7 Macrophage Cells. Molecules 2019, 24(21).

15. El-Bakoush A, Olajide OA. Formononetin inhibits neuroinflammation and increases estrogen receptor beta (ERbeta) protein expression in BV2 microglia. Int Immunopharmacol. 2018;61:325-37.

16. Li W, Sun YN, Yan XT, Yang SY, Kim S, Lee YM, Koh YS, Kim YH. Flavonoids from Astragalus membranaceus and their inhibitory effects on LPS-stimulated pro-inflammatory cytokine production in bone marrow-derived dendritic cells. Arch Pharm Res. 2014;37(2):186-92.

17. Gautam J, Khedgikar V, Kushwaha P, Choudhary D, Nagar GK, Dev K, Dixit P, Singh D, Maurya R, Trivedi R. Formononetin, an isoflavone, activates AMP-activated protein kinase/beta-catenin signalling to inhibit adipogenesis and rescues C57BL/ 6 mice from high-fat diet-induced obesity and bone loss. Br J Nutr. 2017;117(5):645-61.

18. Wang Y, Zhao H, Li X, Wang Q, Yan M, Zhang H, Zhao T, Zhang N, Zhang P, Peng L, et al.

Formononetin alleviates hepatic steatosis by facilitating TFEB-mediated lysosome biogenesis and lipophagy. J Nutr Biochem. 2019;73:108214.

19. Hardie DG, Schaffer BE, Brunet A. AMPK: An Energy-Sensing Pathway with Multiple Inputs and Outputs. Trends Cell Biol. 2016;26(3):190-201.

20. Guma M, Wang Y, Viollet B, Liu-Bryan R. AMPK Activation by A-769662 Controls IL-6 Expression in Inflammatory Arthritis. PLoS One. 2015;10(10):e0140452.

21. Kato K, Tokuda H, Matsushima-Nishiwaki R, Natsume H, Kondo A, Ito Y, Kozawa O, Otsuka T. AMPK limits IL-1-stimulated IL-6 synthesis in osteoblasts: involvement of IkappaB/NF-kappaB pathway. Cell Signal. 2012;24(8):1706-12.

22. Cao L, Zhang X, Yuan S, Cheng K, Zhang X. Autophagy induced by enterovirus 71 regulates the production of IL-6 through the p38MAPK and ERK signaling pathways. Microb Pathog. 2019;131:120-7.

23. Wang Y, Zhu Y, Gao L, Yin H, Xie Z, Wang D, Zhu Z, Han X. Formononetin attenuates IL-1beta-induced apoptosis and NF-kappaB activation in INS-1 cells. Molecules. 2012;17(9):10052-64.

24. Zhou Z, Zhou X, Dong Y, Li M, Xu Y. Formononetin ameliorates high glucoseinduced endothelial dysfunction by inhibiting the JAK/STAT signaling pathway. Mol Med Rep. 2019;20(3):2893-901.

25. Aladaileh SH, Hussein OE, Abukhalil MH, Saghir SAM, Bin-Jumah M, Alfwuaires MA, Germoush MO, Almaiman AA, Mahmoud AM. Formononetin Upregulates Nrf2/HO-1 Signaling and Prevents Oxidative Stress, Inflammation, and Kidney Injury in Methotrexate-Induced Rats. Antioxidants (Basel) 2019, 8(10). 
26. Medina EA, Oberheu K, Polusani SR, Ortega V, Velagaleti GV, Oyajobi BO. PKA/AMPK signaling in relation to adiponectin's antiproliferative effect on multiple myeloma cells. Leukemia. 2014;28(10):2080-9.

27. Kari S, Vasko VV, Priya S, Kirschner LS. PKA Activates AMPK Through LKB1 Signaling in Follicular Thyroid Cancer. Front Endocrinol (Lausanne). 2019;10:769.

28. Tazawa H, Kawaguchi T, Kobayashi T, Kuramitsu Y, Wada S, Satomi Y, Nishino H, Kobayashi M, Kanda $Y$, Osaki M, et al. Chronic inflammation-derived nitric oxide causes conversion of human colonic adenoma cells into adenocarcinoma cells. Exp Cell Res. 2013;319(18):2835-44.

29. Conway EM, Pikor LA, Kung SH, Hamilton MJ, Lam S, Lam WL, Bennewith KL. Macrophages, Inflammation, and Lung Cancer. Am J Respir Crit Care Med. 2016;193(2):116-30.

30. Donath MY, Shoelson SE. Type 2 diabetes as an inflammatory disease. Nat Rev Immunol. 2011;11(2):98-107.

31. Back M, Hansson GK. Anti-inflammatory therapies for atherosclerosis. Nat Rev Cardiol. 2015;12(4):199-211.

32. Esser N, Legrand-Poels S, Piette J, Scheen AJ, Paquot N. Inflammation as a link between obesity, metabolic syndrome and type 2 diabetes. Diabetes Res Clin Pract. 2014;105(2):141-50.

33. Kang S, Tanaka T, Kishimoto T. Therapeutic uses of anti-interleukin-6 receptor antibody. Int Immunol. 2015;27(1):21-9.

34. Carling D. AMPK signalling in health and disease. Curr Opin Cell Biol. 2017;45:31-7.

35. Smith BK, Marcinko K, Desjardins EM, Lally JS, Ford RJ, Steinberg GR. Treatment of nonalcoholic fatty liver disease: role of AMPK. Am J Physiol Endocrinol Metab. 2016;311(4):E730-40.

36. Jiang S, Li T, Ji T, Yi W, Yang Z, Wang S, Yang Y, Gu C. AMPK: Potential Therapeutic Target for Ischemic Stroke. Theranostics. 2018;8(16):4535-51.

37. Steneberg P, LindahI E, DahI U, Lidh E, Straseviciene J, Backlund F, Kjellkvist E, Berggren E, Lundberg I, Bergqvist I, et al: PAN-AMPK activator 0304 improves glucose homeostasis and microvascular perfusion in mice and type 2 diabetes patients. JCI Insight 2018, 3(12).

\section{Figures}




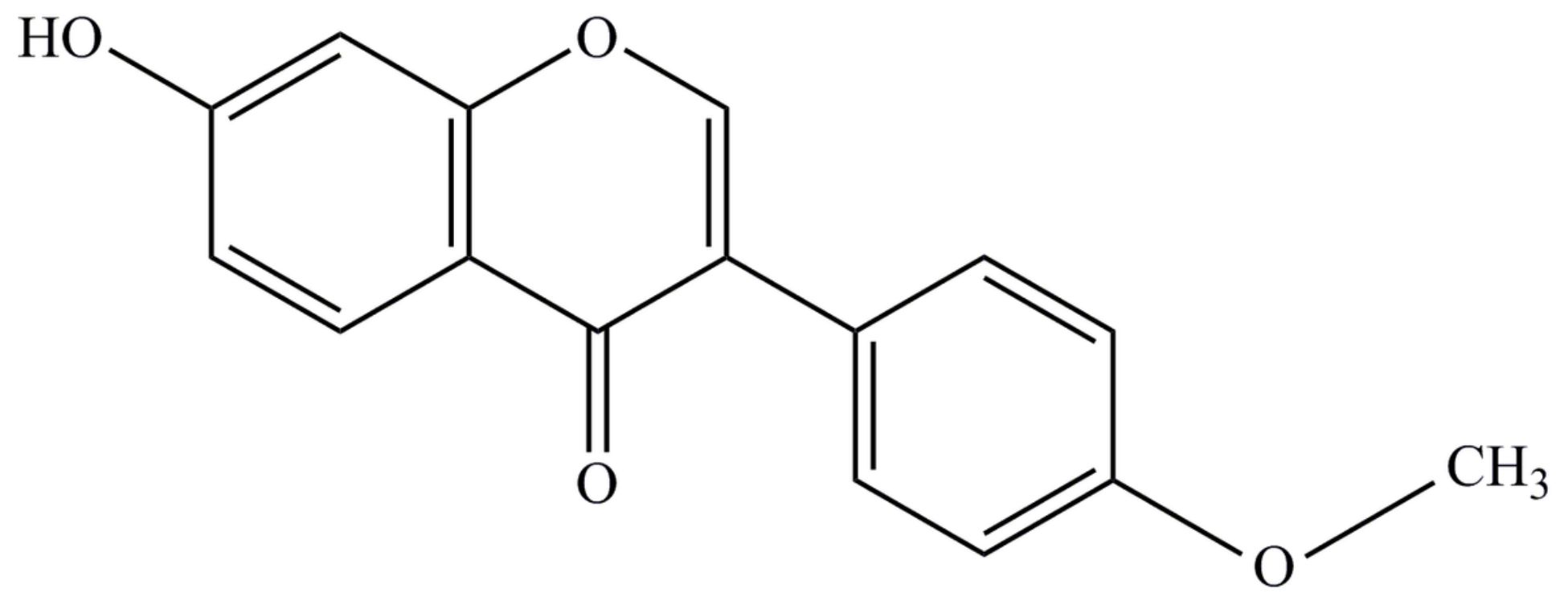

Figure 1

Chemical structure of FMN. 

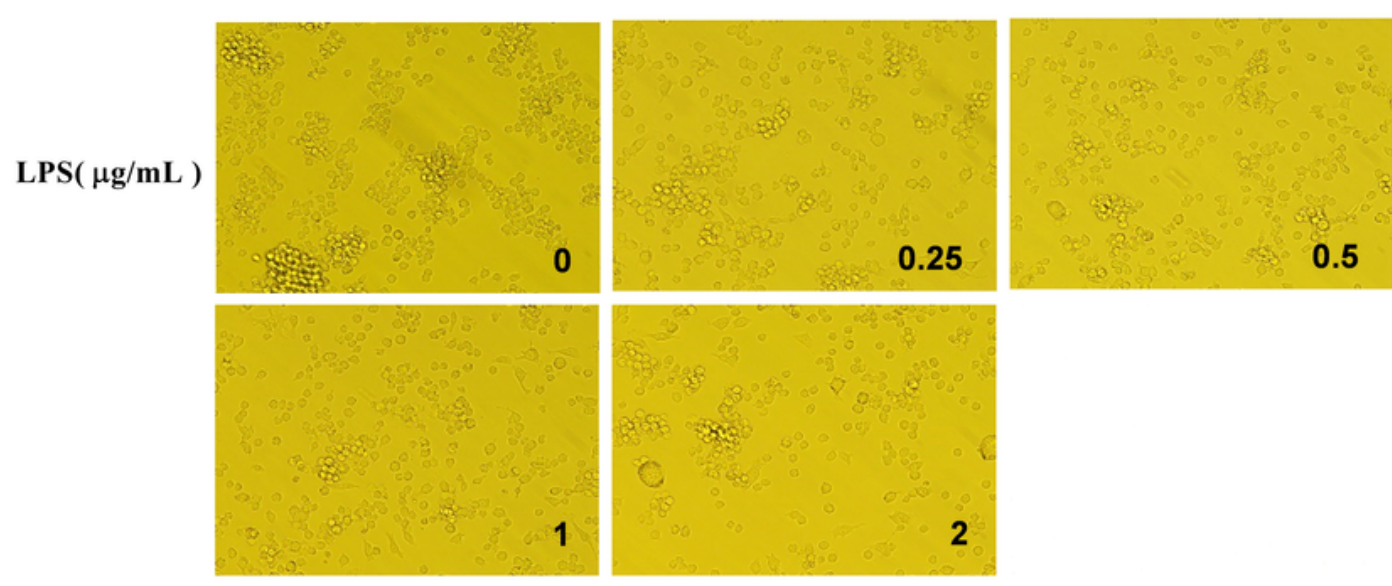

1

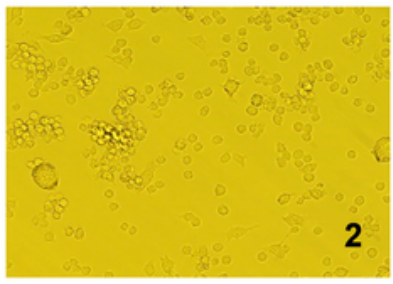

a
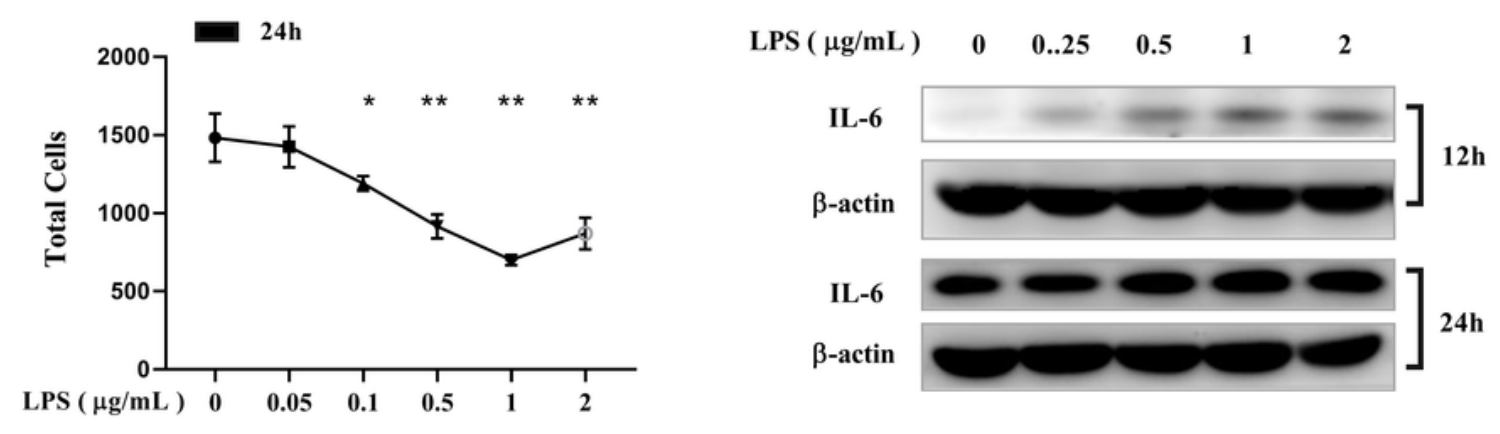

b

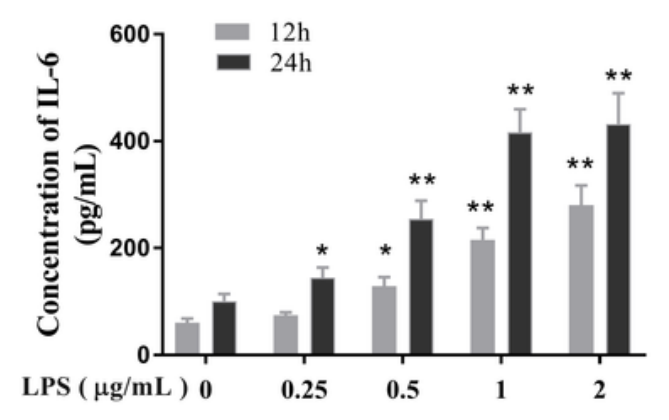

c d

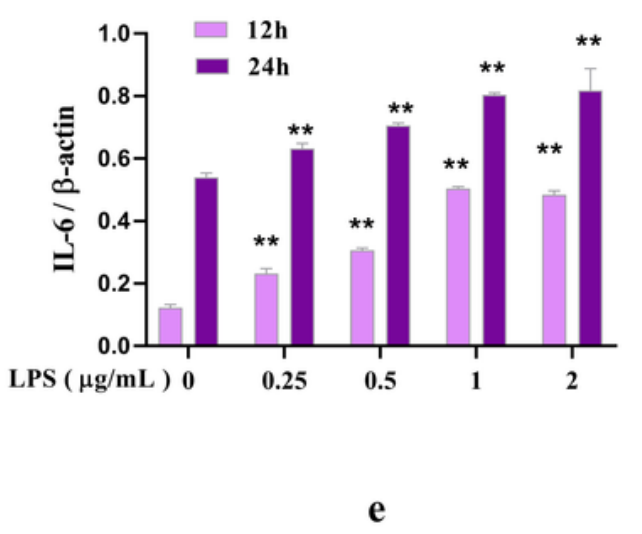

\section{Figure 2}

Effects of LPS on cell count, morphology, and IL- 6 expression in ANA-1 cells. The morphology of ANA-1 cells was observed under an inverted microscope (a). ANA-1 cells were counted using an HCS (b). IL-6 protein expression level in supernatants from cells was assayed via ELISA (c). Western blotting was performed to determine the levels of IL-6 in cells, with $\beta$-actin used as the control $(d, e)$. The numerical 
data were analyzed using the one-way ANOVA and the protein level data were analyzed using the t-test. Data are represented as the mean $\pm S D(n=3)$. ${ }^{*} p<0.05$ and ${ }^{* *} p<0.01$, compared with the control group.

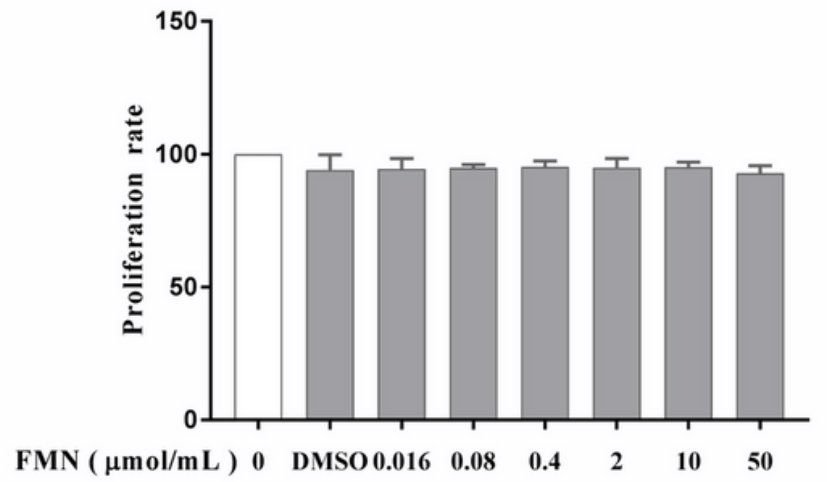

a

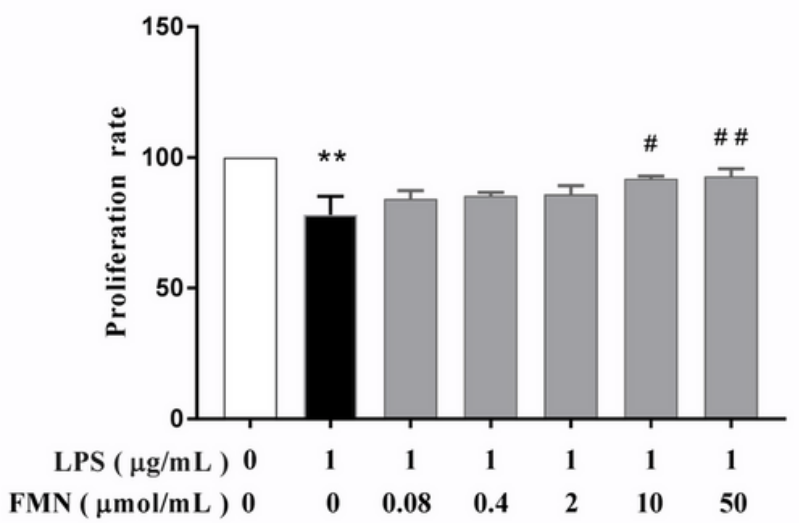

b

Figure 3

Determination of FMN toxicity in ANA-1 cells. The proliferation rate of non-treated ANA-1 cells (a) and LPS-treated cells (b) was determined using the CCK-8 kit. Data were analyzed using the one-way ANOVA. Data are presented as the mean $\pm S D(n=3) .{ }^{*} p<0.01$, compared with the control group. $\# p<0.05$ and $\# \# p<0.01$, compared with the LPS group. 


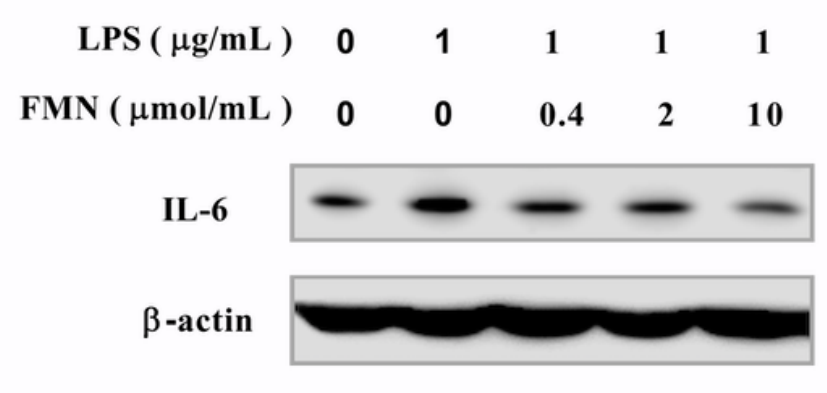

a

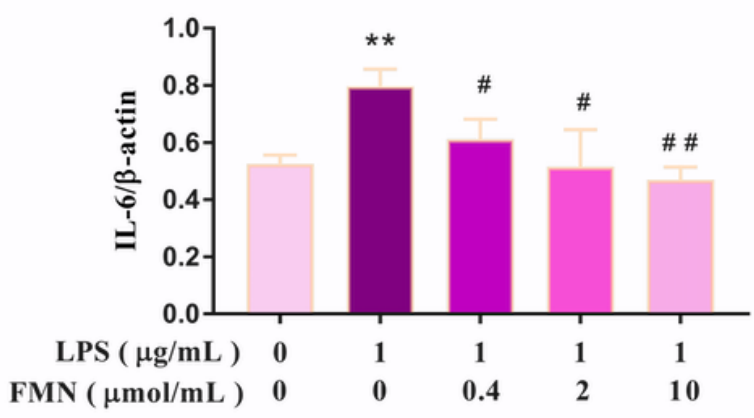

b

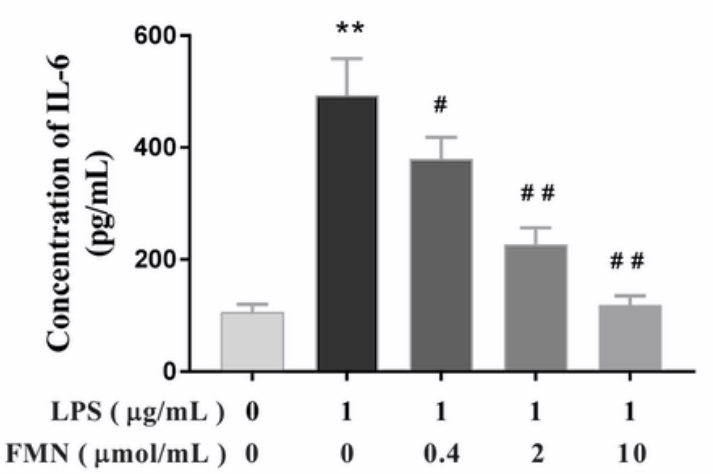

c

Figure 4

Effect of FMN on the expression of IL-6 in LPS-stimulated ANA-1 cells. Western blotting was performed to determine the levels of IL- 6 in cells, with $\beta$-actin used as the control $(a, b)$. IL- 6 protein expression level in supernatants from cells was assayed via ELISA (c). Data were analyzed using the t-test. Data are presented as the mean \pm standard deviation $(n=3)$. ${ }^{\star \star} p<0.01$, compared with the control group. $\# p<$ 0.05 and $\# \# p<0.01$, compared with the LPS group. 

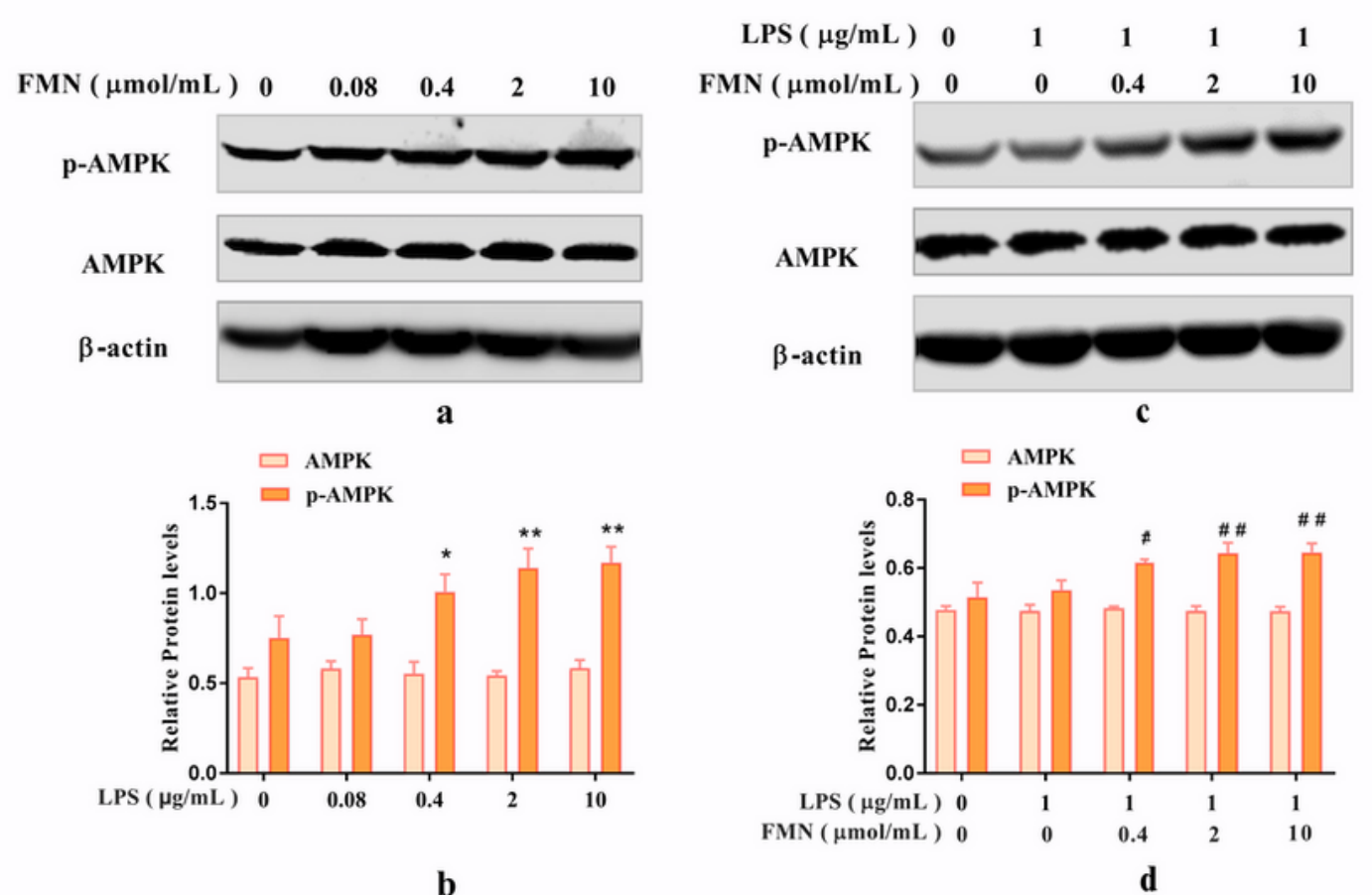

b
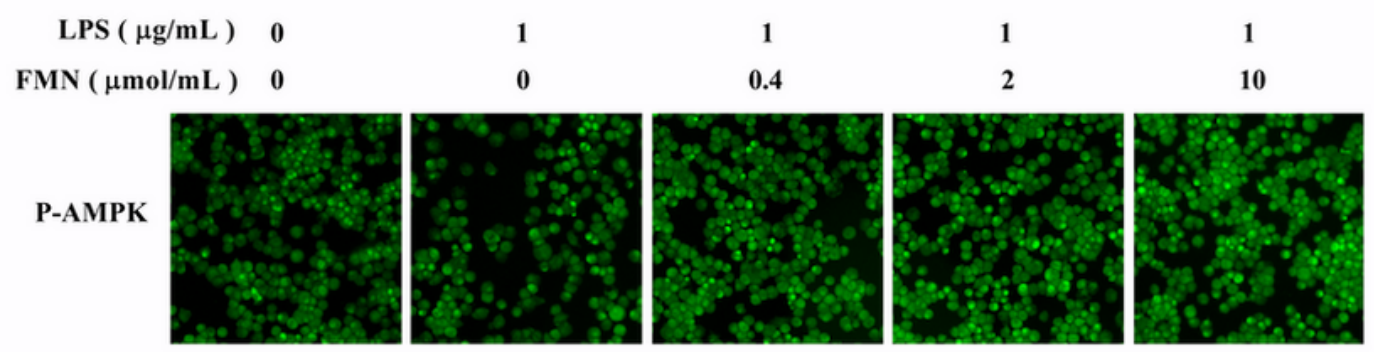

f
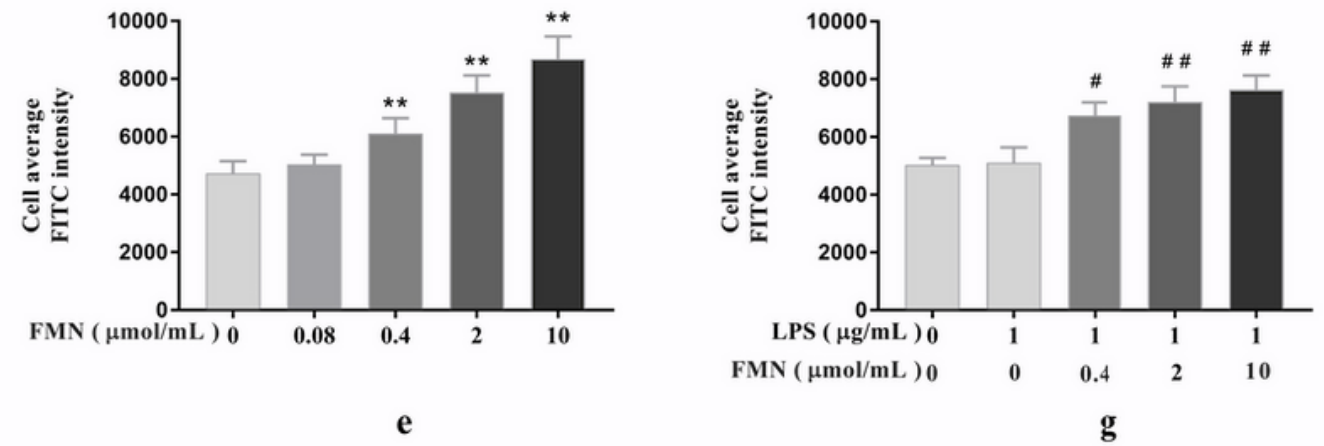

\section{Figure 5}

Effect of FMN on the phosphorylation of AMPK in ANA-1 cells and LPS-treated cells. Western blotting was performed to determine the levels of p-AMPK in cells with $(a, b)$ or without $(c, d)$ LPS treatment, with $\beta$-actin used as the control. The activation of p-AMPK was detected using immunostaining ( $f$ ), and the levels of average FITC intensity of cells with (e) or without (g) LPS treatment were examined using an 
HCS (200x). Data were analyzed using the t-test. Data are presented as the mean $\pm \operatorname{SD}(n=3) .{ }^{*} p<0.05$, compared with the control group. \#p $<0.05$ and \#\#p $<0.01$, compared with the LPS group.

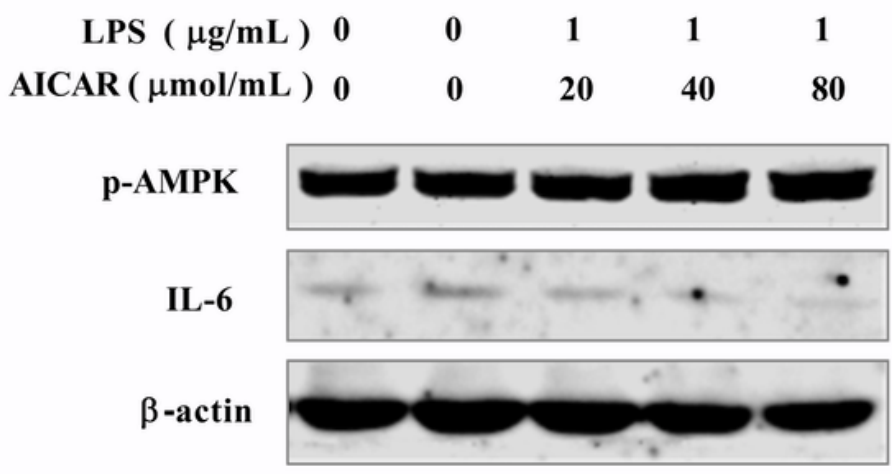

C
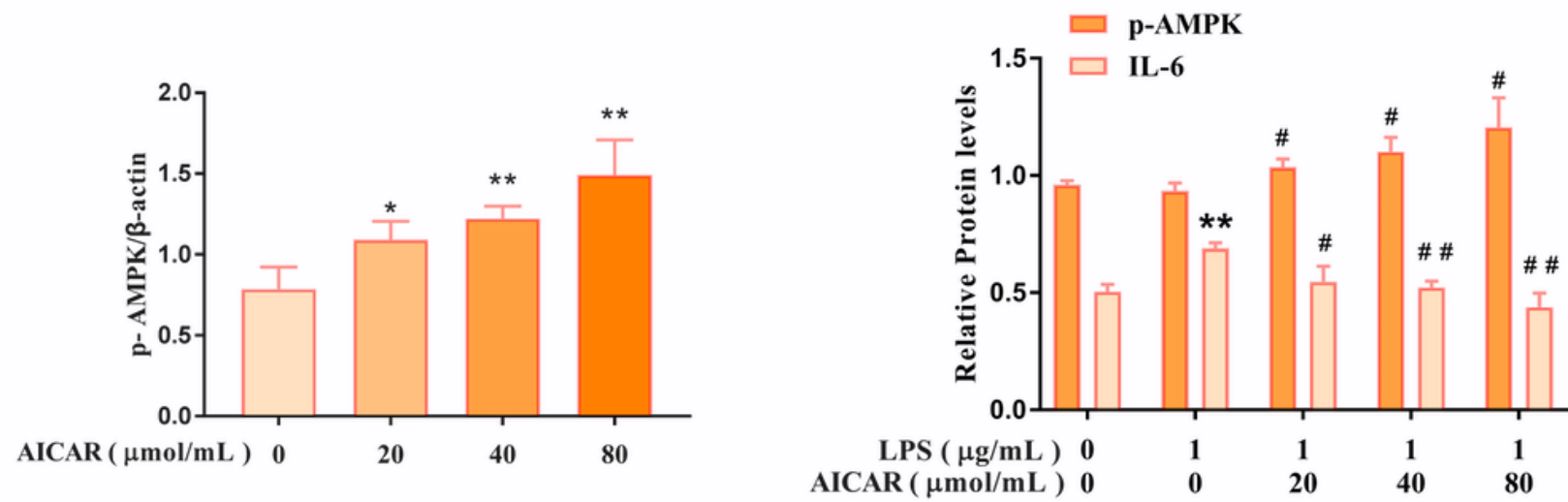

b

d

\section{Figure 6}

Effect of AICAR on p-AMPK and IL-6 in LPS-stimulated ANA-1 cells. p-AMPK was determined by western blotting in AICAR treated ANA-1 cells, with $\beta$-actin used as the control $(a, b)$. Western blotting was performed to determine the levels of IL-6 and p-AMPK caused by co-treatment with different doses of AICAR and LPS in ANA-1 cells, with $\beta$-actin used as the control (c, d). The data were analyzed using a ttest. Data are presented as the mean \pm SD $(n=3)$. ${ }^{*} p<0.05$ and ${ }^{*} p p<0.01$, compared with the control group. \#p $<0.05$ and \#\#p $<0.01$, compared with the LPS group. 


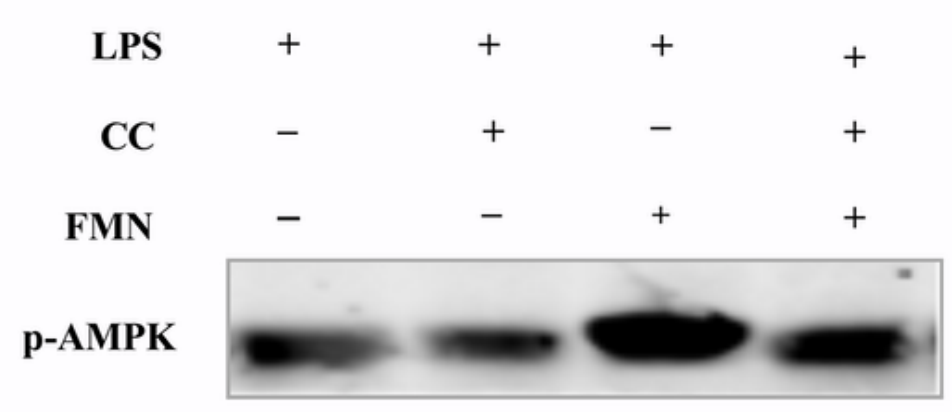

IL-6
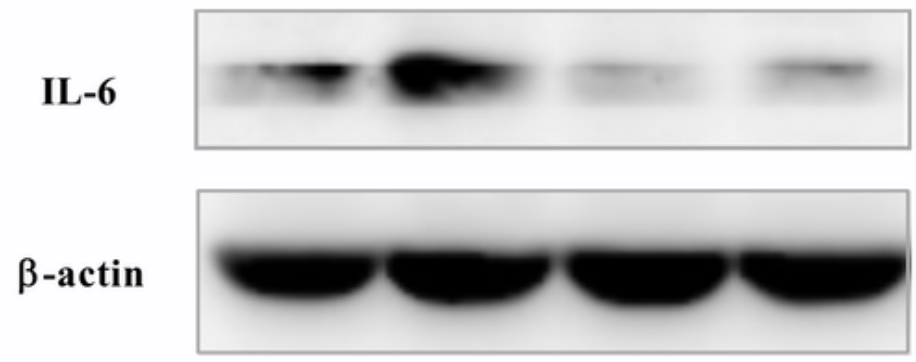

a

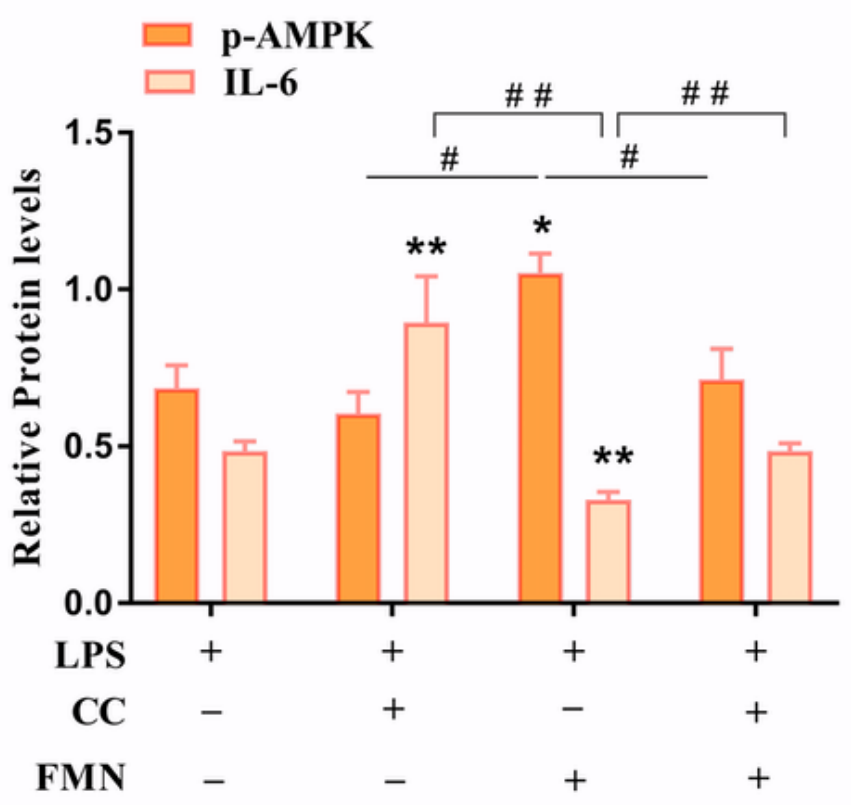

b

Figure 7

Effect of compound $C$ on the phosphorylation of AMPK and IL-6 in ANA-1 cells. Western blotting was performed to determine the effect of compound $C$ on the levels of p-AMPK and IL- 6 in ANA- 1 cells with $\beta$ actin used as the control $(a, b)$. Data were analyzed using the t-test. Data are presented as mean \pm SD ( $n$ = 3). ${ }^{*} p<0.05$ and ${ }^{* \star} p<0.01$, compared with the LPS group. $\# p<0.05$ and $\# \# p<0.01$, compared with different groups. 

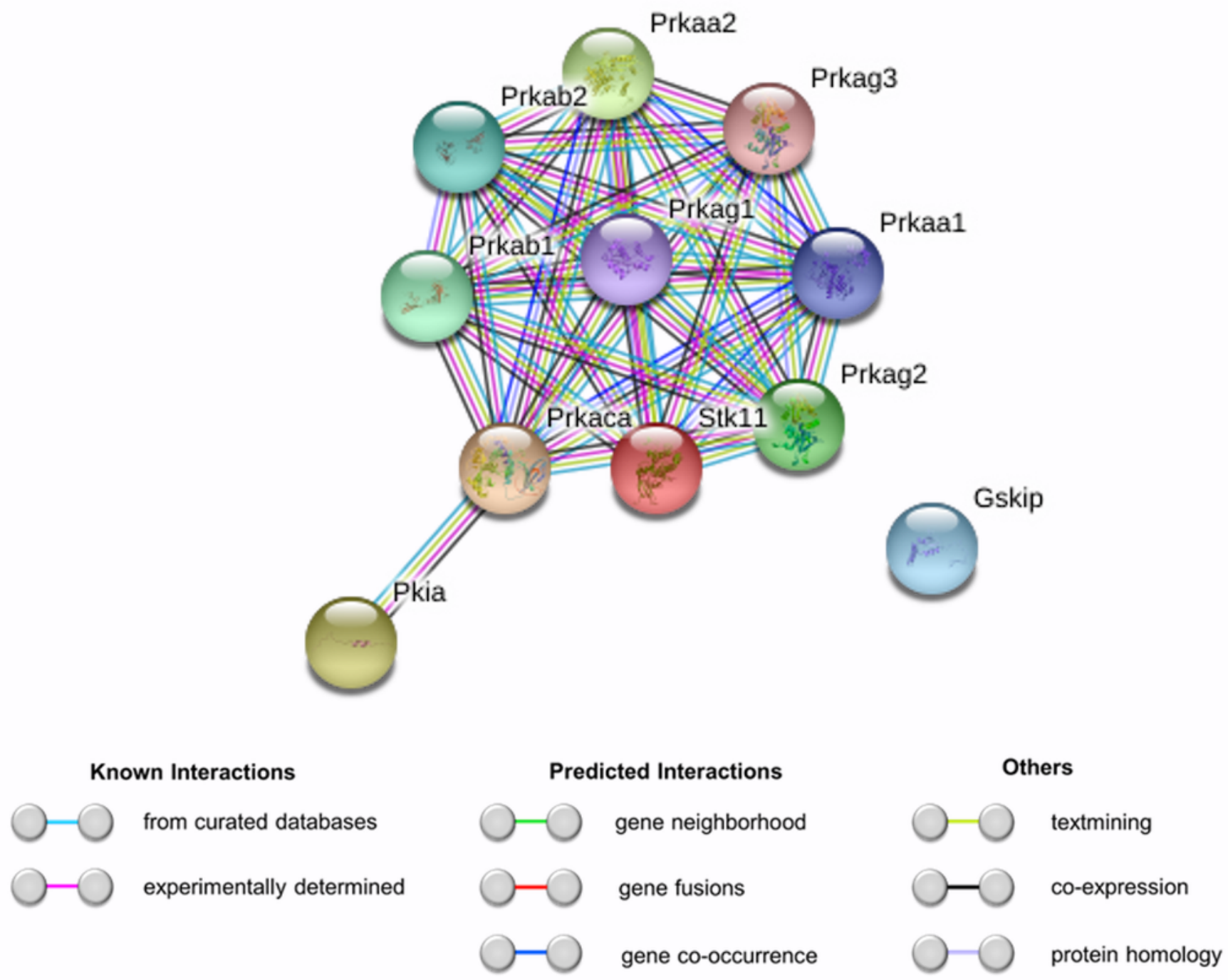

Figure 8

Prediction of the potential targets of FMN. 